Check for updates

Cite this: RSC Adv., 2017, 7, 30205

Received 12th April 2017

Accepted 31st May 2017

DOI: $10.1039 / \mathrm{c7ra04138a}$

rsc.li/rsc-advances

\section{Design and development of caffeic acid conjugated with Bombyx mori derived peptide biomaterials for anti-aging skin care applications $\dagger$}

\author{
Hoomin Lee, $\mathbb{D} \ddagger^{\mathrm{a}}$ A. T. Ezhil Vilian, $\dot{t}^{\mathrm{b}}$ Jun Yeong Kim, $\dot{+}^{\mathrm{c}}$ Myeung Hwan Chun, \\ Jung Sang Suh, (DD ${ }^{d}$ Hyo Hyun Seo, ${ }^{c}$ Seung Hee Cho, ${ }^{c}$ II Soo Shin, ${ }^{e}$ Sung Jun Kim, ${ }^{e}$ \\ Seok Hoon Park, ${ }^{f}$ Young-Kyu Han, (D) ${ }^{* b}$ Jeong Hun Lee ${ }^{* c}$ and Yun Suk Huh (D)*ag
}

Over the last few centuries, aging-related diseases have become a part of the global health care problem. The skin is essentially more sensitive to aging because it is influenced by environmental exposure and genetic (genetically determined) processes. The use of a suitable cosmetic anti-aging material using a biomolecule with conventionally important medicine can help to protect damaged skin tissue. A new topical cosmetic anti-aging material should simulate the function of an extracellular matrix with its enhanced physiochemical, biological oxidative stress, and antioxidant properties. This study examined the significant features of CA-APPPKK (caffeic acid conjugated with peptide (APPPKK)) to determine if it meets the requirements for a cosmetic anti-aging biomolecule. Non-toxic CA-APPPKK was synthesized using a simple approach and CA stabilized with a peptide to form the new biomaterials. The cytotoxicity of the CA-APPPKK was examined with a CCK- 8 assay and a reactive oxygen species (ROS) assay to evaluate the biological applications. CA-APPPKK caused a decrease in cell viability and membrane damage, and the degree of induction was dose dependent. The longevity-extending role of CA-APPPKK was attributed to its anti-oxidative activity and increased oxidative stress resistance. The antiinflammatory capacity of CA-APPPKK was measured using a NO assay. This new approach indicates that CA-APPPKK is a potentially cosmetic anti-aging biomaterial for the prevention of oxidative stressinduced premature skin ageing and can be used to study aging-related diseases.

\section{Introduction}

Aging of the skin is a complex biological process comprising two features: intrinsic aging and photoaging influenced by both environmental (sun radiation, oxidative stress and pollution) and genetic (genetically determined) processes. ${ }^{1,2}$ In particular, the extreme initiation of reactive oxygen species (ROS) can

${ }^{a}$ Department of Biological Engineering, Biohybrid Systems Research Center (BSRC), Inha University, Incheon 22212, Republic of Korea. E-mail: yunsuk.huh@inha.ac.kr ${ }^{b}$ Department of Energy and Materials Engineering, Dongguk University-Seoul, Seoul 04620, Republic of Korea. E-mail: ykenergy@dongguk.edu

${ }^{c}$ Anti-aging Research Institute of BIO-FD\&C Co. Ltd., Incheon 22212, Republic of Korea. E-mail: jhlee@biofdnc.com

${ }^{d}$ Department of Chemistry, Seoul National University, Seoul, 151-747, Republic of Korea

${ }^{e}$ Research Institute of Charming Cosmetics Co. Ltd., Incheon 22212, Republic of Korea Industry-Academic Cooperation Foundation, Kangnam University, Yongin-si, 16979, Republic of Korea

${ }^{g}$ WCSL of Integrated Human Airway-on-a-Chip, Inha University, Incheon, Republic of Korea

$\dagger$ Electronic supplementary information (ESI) available. See DOI: $10.1039 / \mathrm{c} 7 \mathrm{ra04138a}$

\$ Three authors equally contributed. generate a significant decrease in metabolic function (senescence, apoptotic or necrotic) or cell death, and their presence inside the cell can lead to skin aging. ${ }^{3}$ Reactive oxygen species play an essential role in initiating the cytoplasmic signal transduction pathways in the tenant fibroblasts and assist in cell growth, differentiation, senescence, and connective tissue degradation, as well as affecting permanent genetic modification. ${ }^{4}$ Consequently, antioxidant therapy may be a suitable way to reduce the harmful causes of reactive oxygen species and protect the skin from aging. ${ }^{5}$

Caffeic acid (CA, 3,4-dihydroxycinnamic acid) is a naturally occurring flavonoid molecule found at high concentrations in several plants in many forms, such as glycosides, esters and the free form. ${ }^{6-8}$ The compound is present in various plantderived foods, including fruit, black tea, coffee, olive oil, red, and white wines. ${ }^{9}$ As with other flavonoids, CA has been used widely for various biological activities includes anti-bacterial and antiviral effects, anti-allergic and anti-coagulatory effects, antioxidant and anti-inflammatory activities, antimutagenic and anticancer effects and clinical applications because of its biodegradability, biocompatibility, low allergenic properties, and low toxicity. ${ }^{10}$ Recently, some research groups around the world have been modifying the profile of CA 
and reported that some CA products have diverse biological activities. $^{\mathbf{1 1}}$

Bioactive peptides have positive effects in human health and connected functions, including antioxidant activity, antimicrobial activity, angiotensin-I converting enzyme inhibition, and immune modulation. The prepared bioactive peptides have many potential applications in a wide areas, such as cosmetics, food flavors, nutraceuticals, and pharmaceuticals. ${ }^{\mathbf{1 2}}$ Moreover, bioactive compounds have been studied and used in bio-related areas owing to their excellent structural and biological properties. Bioactive peptides have been applied in many clinical areas over the last 20 years. The active component of caffeic acid contains two vicinal hydroxyl groups on the aromatic ring, known as the catechol group, which imparts stability, surface charges, and allows interactions of the peptide with other molecules. ${ }^{13}$ The nature of the capping agent affects both the physical properties and biological properties of the peptide. ${ }^{\mathbf{1 4}}$ The development of eco-friendly and multifunctional caffeic acid-APPPKK biomaterials as free radical scavengers to prevent oxidative damage and reduce toxicity has attracted increasing attention. ${ }^{15}$ Owing to their antioxidant activity, CA-based peptide biomaterials have been used in a wide range of consumer manufacture, ranging from cosmetics to household products, as well as clinical applications, including imaging, drug delivery and disinfection. ${ }^{\mathbf{1 6 , 1 7}}$ Furthermore, CA-incorporated, APPPKK-impregnated catheters and wound dressings have been applied in the therapeutic applications of tissue engineering and drug delivery. ${ }^{18}$ On the other hand, earlier studies have reported that biomaterials have size-, time- and dose dependent cytotoxicity, where smaller size nanomaterials with a longer exposure time at higher concentrations have the highest toxicity. ${ }^{19,20}$ A possible mechanism of the cytotoxicity of caffeic acid-conjugated APPPKK involves a disruption of the mitochondrial respiratory chain, increase in ROS production, and an interruption of ATP synthesis, which in turn causes the oxidation of lipids, proteins and DNA, leading to many disease conditions. ${ }^{21}$ To the best of the authors' knowledge, this is the first report of the preparation of CA-conjugated APPPKK biomaterials to assess their potential applications in skin tissue engineering and pharmaceutical industry.

A facile, efficient and economical route has been developed to synthesize CA-APPPKK biocompatible materials using APPPKK assisted with CA. CA-APPPKK was characterized by matrix assisted laser desorption/ionization time of flight (MALDI-TOF) and liquid chromatography-mass spectrometry (LC-MS) and the effects of the exposure dose affecting the cell response and cytotoxicity of the prepared CA-APPPKK in human fibroblast cell were studied. The level of toxicity was evaluated using the changes in cell morphology, cell viability and oxidative stress studies. Therefore, the ROS scavenging activity in the skin is important for anti-ageing activity. The CAAPPPKK biomaterials exhibited good fastness properties as well as excellent antioxidant activity. In addition, they played an important role in extending lifespan, and improving oxidative stress resistance. CA supports good antioxidant property. The antioxidant activities were studied using a DPPH antioxidant assay.

\section{Materials and methods}

\subsection{Materials}

Fmoc-Ala-OH, Fmoc-Lys (Boc)-OH, and Fmoc-Pro-OH were purchased from Bead-Tech (Seoul, Korea). Fmoc-Lys (Boc)Wang resin was obtained from Merck (Germany). Hydroxybenzotriazole (HOBt) and $N, N^{\prime}$-diisopropylcarbodiimide (DIC) were acquired from GLS (Shanghai, China). Caffeic acid was supplied by 4Chem (Suwon, Korea). Dichloromethane (DCM), dimethylformamide (DMF), and piperidine were procured from Daejung (Siheung, Korea).

\subsection{Synthesis of APPPKK and caffeoyl-APPPKK}

Fmoc-Lys (Boc)-Wang resin was swelled in dichloromethane (DCM, $5 \mathrm{~mL}$ ) for $30 \mathrm{~min}$. The resin was filtered and washed with dimethylformamide (DMF). After deprotonation of Fmoc with $20 \%$ piperidine in DMF for $20 \mathrm{~min}$ or $15 \mathrm{~min}$, Fmoc-Lys (Boc)$\mathrm{OH}$ (3.0 eq.), HOBt (3.0 eq.), and $N, N^{\prime}$-diisopropylcarbodiimide (DIC) (3.0 eq.) were added to a $\mathrm{NH}_{2}$-Lys (Boc)-Wang resin (Sub. $0.7 \mathrm{mmol} \mathrm{g}^{-1}, 0.06 \mathrm{mmol}$ ) in DMF. The amino acids were then coupled to the resin by the general HOBt-DIC mediated solid-phase peptide synthesis (SPPS) protocol. After the coupling of the final CA, the resin was filtered, washed, and dried under high vacuum. Finally, the resin was treated with reagent $\mathrm{K} \quad$ (TFA : phenol : $\mathrm{H}_{2} \mathrm{O}$ : thioanisole : EDT = $82.5: 5: 5: 5: 2.5 \%)$ for $3 \mathrm{~h}$. The resin was filtered and washed with DMF and DCM. The collected filtrate was precipitated with pre-cooled ether to give a crude peptide.

Analytical reverse phase high performance liquid chromatography (RP-HPLC) was performed on a Waters $1525 \mathrm{u}$ Binary HPLC Pump with a Shimpack RP-C18 column $(250 \times 4.6 \mathrm{~mm}$ ID; $5 \mu \mathrm{m}$ particle size, Shimadzu). The solvent system consisted of $0.1 \%$ TFA in $\mathrm{H}_{2} \mathrm{O}$ (solvent A) and $0.1 \%$ TFA in acetonitrile (solvent B). Elution was achieved by applying a linear gradient

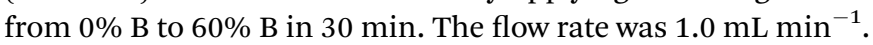
The peptide peaks were detected with a Waters 996 Photodiode Array Detector.

Semi-preparative RP-HPLC was performed on a Waters HPLC system (pump 600E, detector UV-484). A Gemini RP-C18 column $(250 \times 21.2 \mathrm{~mm}$ ID; $5 \mu \mathrm{m}$ particle size, Phenomenex $)$ was used. The solvent system consisted of $0.1 \%$ TFA in $\mathrm{H}_{2} \mathrm{O}$ (solvent A) and $0.1 \%$ TFA in acetonitrile (solvent B). Elution was achieved by applying a linear gradient from $5 \%$ to $15 \%$ B in $15 \mathrm{~min}$. The

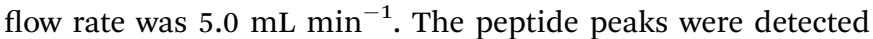
spectrophotometrically $(\lambda=230 \mathrm{~nm})$.

The molecular weight of the synthesized peptide was analyzed by MALDI-TOF (Voyager, used matrix: $\alpha$-cyano- 4 hydroxy cinnamic acid (CHCA)) mass spectrometry.

The Fourier transform infrared (FT-IR) spectra were acquired using FT/IR-6600, (JASCO). 


\subsection{Cell culture and viability assay}

The human fibroblast cell line (CCD-1064SK, ATCC, USA) was maintained in Dulbecco's Modified Eagles' Media (DMEM; 11965-092, Gibco, USA) supplemented with 10\% fetal bovine serum (FBS; 16000044, Gibco, USA) and 1\% penicillinstreptomycin (15070063, Gibco, USA). The cells were incubated at $37^{\circ} \mathrm{C}$ and $5 \% \mathrm{CO}_{2}$. The cell viability after exposure to the different CA-APPPKK was determined using a Cell Counting Kit-8 (CCK-8) assay (CK04-11, Dojindo, Japan). Fibroblast cells were seeded in 12 -well cell plates at $1.0 \times 10^{4}$ cells per well and incubated for 24 hours at $37{ }^{\circ} \mathrm{C}$ and $5 \%$ $\mathrm{CO}_{2}$. After $24 \mathrm{~h}$ of cell attachment, the cells were incubated with different concentrations of CA, APPPKK, and CA$\operatorname{APPPKK}(1,10,50$ and $100 \mu \mathrm{M})$ for $24 \mathrm{~h}$. The samples were removed and the cells were washed three times with PBS. A $10 \mu \mathrm{L}$ sample of CCK-8 dye and $100 \mu \mathrm{L}$ of Dulbecco's Modified Eagle's Medium (DMEM) cell culture media was added to each well and incubated for $2 \mathrm{~h}$ at $37^{\circ} \mathrm{C}$. This step was performed to avoid any unwanted scattering from the particles during the absorbance measurements. The absorbance of the supernatant was collected at $420 \mathrm{~nm}$ using a SpectraMax Plus 384 microplate reader. The percentage relative cell viability was calculated by normalizing the treated cells to that of the untreated control sample. The medium and MTS reagent without the cells served as a method blank for all samples.

\subsection{Detection of reactive oxygen species (ROS) production}

2.4.1. Detection of ROS in chemical level. A $100 \mu \mathrm{L}$ sample of $10 \mu \mathrm{M}$ DCF-DA (DCF-DA 287810, MERCK MILLIPORE, German) was reacted with $0.01 \mathrm{~N} \mathrm{NaOH} 4 \mathrm{~mL}$ for $30 \mathrm{~min}$ to achieve complete deacetylation in the darkroom. The mixture solution was then neutralized with PBS $20 \mathrm{~mL}$. Each sample (1, 10,50 , and $100 \mu \mathrm{M}$ ) in PBS with DCF-DA was mixed with HRP (P8375, SIGMA, USA) (2.2 unit per $\mathrm{mL}$ ) at a $1: 1$ volumetric ratio and reacted in a darkroom for $30 \mathrm{~min}$. Centrifugation proceeded at $13000 \mathrm{rpm}$ and $4{ }^{\circ} \mathrm{C}$ for $10 \mathrm{~min}$. The supernatant solution was moved to a 96 well black plate and the fluorescence intensity of DCF was observed with excitation and emission wavelengths at $485 \mathrm{~nm}$ and $535 \mathrm{~nm}$, respectively. The standard curve was obtained from a $\mathrm{H}_{2} \mathrm{O}_{2}$ solution.

2.4.2. Detection of ROS in cell level. The generation of ROS was evaluated using 2',7-dichlorodihydrofluorescein diacetate $\left(\mathrm{H}_{2} \mathrm{DCFDA}\right.$, Invitrogen) staining. In the presence of horseradish peroxidase and $\mathrm{H}_{2} \mathrm{O}_{2}$, DCF-DA is converted to highly fluorescent $2^{\prime}, 7^{\prime}$-dichlorodihydrofluorescein (DCF). The ROS assay was performed according to the supplier's instructions. The confluent cells incubated with different concentrations $(6.5,62.5,125,250 \mu \mathrm{M})$ of CA, APPPKK, and CA-APPPKK for $24 \mathrm{~h}$ in 12-well cell plates were treated with $1 \mathrm{mM}$ of $\mathrm{H}_{2} \mathrm{O}_{2}$ for $30 \mathrm{~min}$. The cells were twice washed with PBS and incubated with $10 \mu \mathrm{M}$ DCF-DA for $40 \mathrm{~min}$ at $37^{\circ} \mathrm{C}$ in the dark. The cells were then washed twice with PBS and analyzed by a microplate reader at an excitation and emission wavelength of $485 \mathrm{~nm}$ and $530 \mathrm{~nm}$, respectively.

\subsection{Determination of antioxidant activity}

A $100 \mu \mathrm{L}$ sample of DPPH (2,2-diphenyl-1-picrylhydrazyl, D9132, Sigma, USA) at a concentration of $300 \mu \mathrm{M}$ and $100 \mu \mathrm{L}$ of APPPKK, CA-APPPKK, and L-ascorbic acid (10300S0301, JUNSEI, Japan) diluted in distilled water to a specific concentration $(1,10,50,100 \mu \mathrm{M})$ was mixed. The resulting solution was reacted in a dark room at $37^{\circ} \mathrm{C}$ for 30 minutes. The solution was measured at $517 \mathrm{~nm}$ using a multi plate reader (Varioskan LUX, Thermo Scientific, USA).

\subsection{Measurement of NO assay}

Macrophage (RAW264.7, KCLB, Korea) cells were cultured in complete DMEM (12100046, Gibco, USA), as described by Chen and Kitts (2015). Briefly, Raw 264.7 cells were seeded on a 12well cell culture plate (30012, SPL, Korea) at a density of $10^{5}$ cells per well in complete DMEM and incubated at $37^{\circ} \mathrm{C}$ overnight. The cells were pre-treated with the samples in fresh DMEM without FBS for $1 \mathrm{~h}$ followed by a treatment with $1.5 \mathrm{~g} \mathrm{~L}^{-1}$ of sodium bicarbonate, 10\% FBS (16000044, Gibco, USA) and 1\% penicillin-streptomycin (15070063, Gibco, USA) for a further $24 \mathrm{~h}$ to induce NO. The cell culture supernatant $(100 \mu \mathrm{L})$ was reacted with $100 \mu \mathrm{L}$ of Griess reagent to form a pink color, which was measured at $517 \mathrm{~nm}$ using a multi plate reader (Varioskan LUX, Thermo Scientific, USA). ${ }^{22}$ Sodium nitrite was used as the standard.

\section{Results and discussion}

In the present study, we have studied that caffeic acid conjugated with APPPKK (yield 30.2\%) having anti-aging effect though oxidant stress prevention. Fig. 1 shows the schematic procedure for the fabrication and application of caffeoylAPPPKK.

\subsection{Characterization studies of CA-APPPKK}

This study evaluated the usefulness of MALDI Q-TOF data for protein identification. Here, a direct determination was performed using a MALDI Q-TOF tandem mass spectrometer. As shown in Fig. 2a and b, the measured $\mathrm{m} / \mathrm{z}$ values were in good agreement with the calculated masses. Fig. $2 \mathrm{a}$ and $\mathrm{b}$ shows the mass spectrum with a distinct peak at $\mathrm{m} / \mathrm{z}$ 638.16, revealing the presence of an APPPKK-like peptide. In addition, the peaks at mass 799.80 are possibly due to the presence of a trace amount $(0.05 \%)$ of a very closely related and structurally similar flavonoid. The high intensity peaks of $\mathrm{m} / \mathrm{z} 799.80$ corresponding to the CA-APPPKK confirmed the conclusion of $\mathrm{Q}$ in and Chait. This suggests that the immonium ions at $m / z 799.80$ is a good indicator for CA-APPPKK. ${ }^{23}$

High-performance liquid chromatography (HPLC) coupled with a high-resolution quadrupole time-of-flight mass spectrometer (Q-TOF) was used. This technique has advantages over other LC methods because of its high sensitivity and selectivity. Fig. 2c and d shows the LC-MS spectra of the individual compounds. The mass peaks observed in the LC-MS spectra at $m / z=319.50$ correspond to APPPKK and at $\mathrm{m} / z=400.50$ correspond to CA. Therefore, the high intensity peak is 


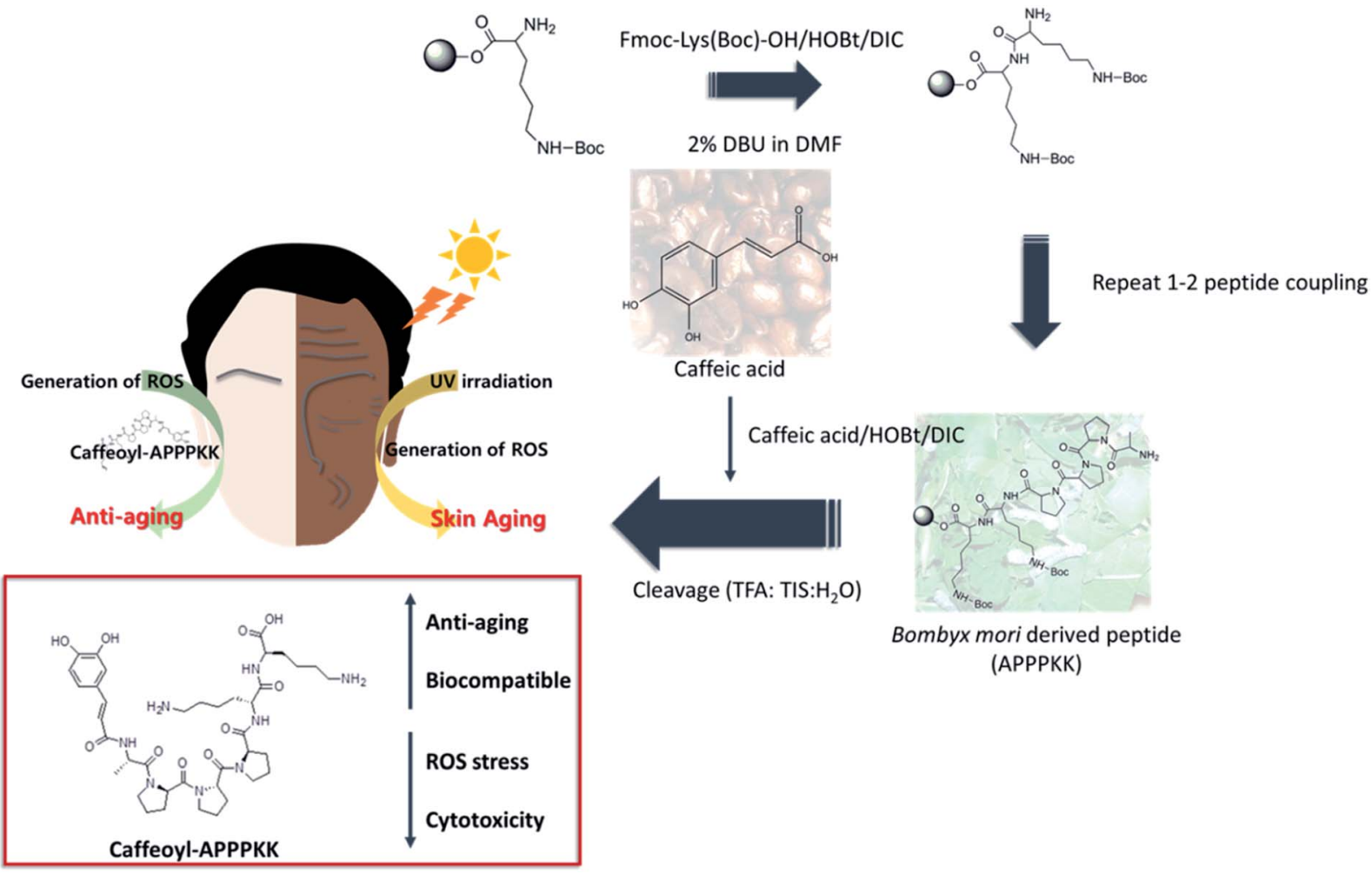

Fig. 1 Schematic procedure for the fabrication and application of caffeoyl-APPPKK.

(a)

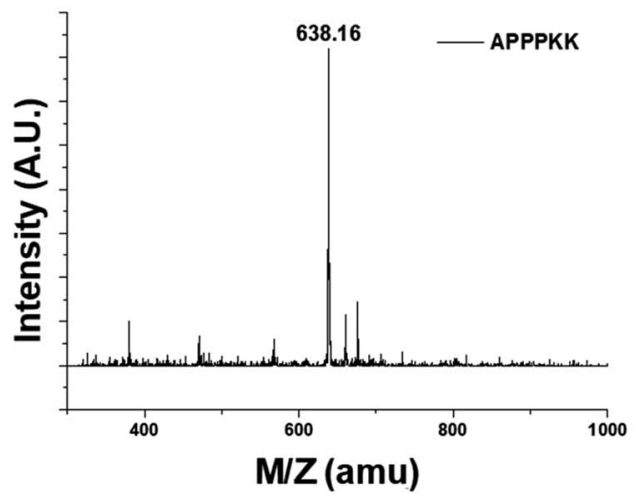

(c)

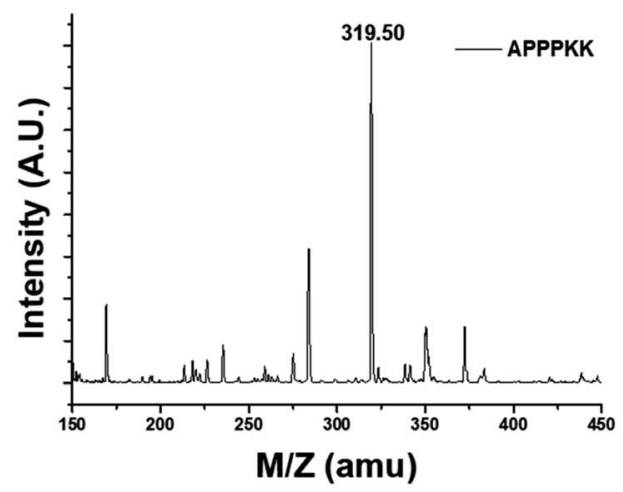

(b)

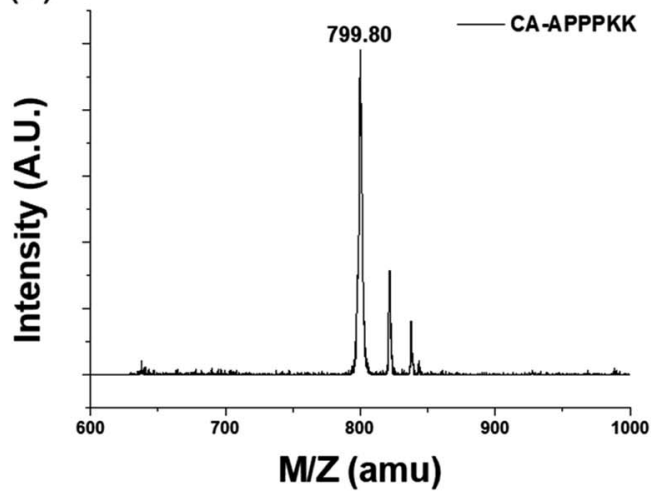

(d)

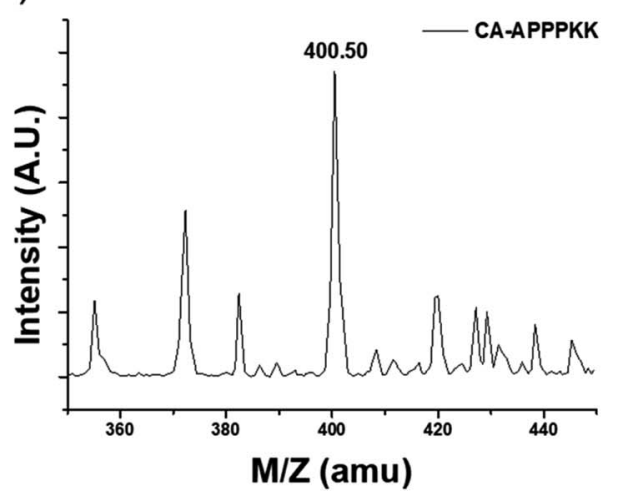

Fig. 2 MALDI-TOF spectrum of (a) APPPKK, (b) CA-APPPKK and (c and d) LC-MS spectrum of the (c) APPPKK, and (d) CA-APPPKK. 
identified as CA-APPPKK. In addition, the compounds were confirmed by comparing the Fig. $2 \mathrm{c}$ and $\mathrm{d}$ with the corresponding LC-MS spectra of the standard. ${ }^{24}$

The modification of CA with APPPKK was observed during the synthesis of the CA-APPPKK by quadrupole-time of flight (TOF) mass spectrometry. The peptide fragment ions obtained from doubly charged precursors produced by nanoelectrospray were mainly y-type ions with some b-ions in the lower mass range. The ratio and intensity of these fragment ions was found to be strongly sequence dependent for MALDI generated ions. The singly charged peptides generated by MALDI showed the preferential cleavage of the C-terminal bond of acidic residues CA and the N-terminal bond of APPPKK. As expected, the quadrupoletime of flight (TOF) mass spectrum of CA-APPPKK showed two additional peak series ( $\mathrm{b}$, and $\mathrm{y}$ ). Their calculated masses were in good agreement with the experimental data, as shown in Fig. 3a. In the high mass region, some unresolved broad signals appear, but are not interpretable in terms of providing sequence-specific information. A possible readout consists of ions that are annotated as b1 to b6, which would give rise to the sequence tag CA, CA-A, CA-AP, CA-APP, CA-APPP, and CA-APPPK. The excellent signal-to-noise ratio and notable observation of the nonpreferential cleavage product ions y1 to y6 caused the further exploration of low intensity signals in the spectrum of doubly charged K, KK, PKK, PPKK, PPPKK, and APPPKK. Additional

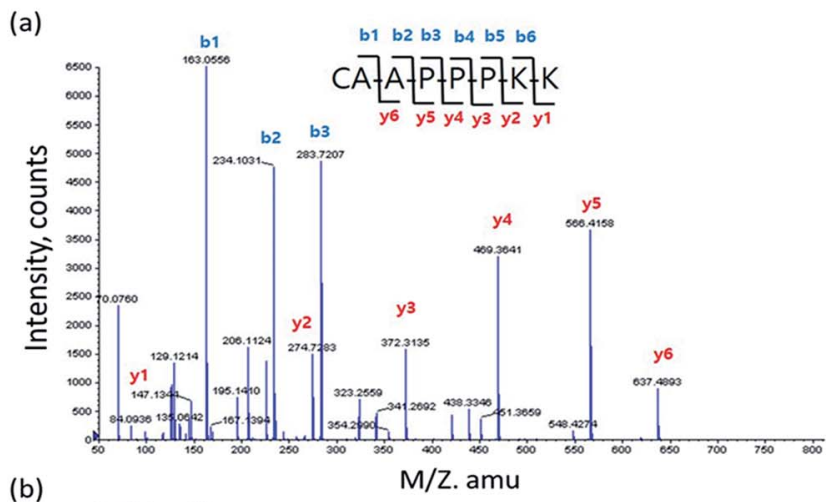

(b)

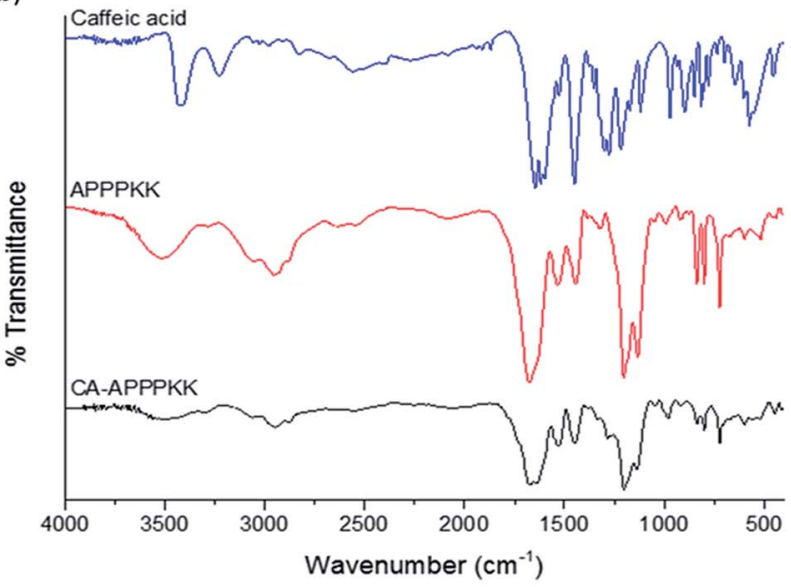

Fig. 3 (a) Quadrupole-time of flight (TOF) spectrum of CA-APPPKK, (b) FTIR spectra of CA, APPPKK, and CA-APPPKK. sequence information might be obtained if sufficient numbers of doubly charged CA-APPPKK precursor ions can be generated. The observation of those peaks provides sufficient information to obtain a sequence tag and identify the CA-APPPKK peptide.

The FTIR spectrum of the prepared APPPKK, CA and CAAPPPKK are presented in Fig. 3b. The FTIR spectra of pure CA, APPPKK and CA-APPPKK were also measured for comparative analysis. The pure CA sample shows strong signals at $3424 \mathrm{~cm}^{-1}$ (-O-H stretching vibration), $3240 \mathrm{~cm}^{-1}$ (benzene ring $=\mathrm{C}-\mathrm{H}$ stretching vibration), $1523 \mathrm{~cm}^{-1}$ (benzene ring $\mathrm{C}=\mathrm{C}$ stretching vibration), $1221 \mathrm{~cm}^{-1}$ (C-OH stretching vibration of phenol), 1642 and $1620 \mathrm{~cm}^{-1}$ (antisymmetry and symmetrical stretching vibrations $\mathrm{C}=\mathrm{O}$ in the ester group $(\mathrm{O}=\mathrm{C}-\mathrm{O}))$. The above result confirms the structure of caffeic acid. ${ }^{25}$ Both APPPKK and CAAPPPKK exhibited signals at 1600-1689, 1479-1573, 1226-1303, and $640-800 \mathrm{~cm}^{-1}$. We observed that the FTIR spectra of CA and CA-APPPKK show the disappearance of the $\mathrm{O}-\mathrm{H}$ vibration attached to the benzene ring and the formation of a new broad signal at $3394 \mathrm{~cm}^{-1}$. Previous report according to that the $\mathrm{H}$ bonded $\mathrm{OH}$ stretch normally occurred as broad spectra in the region $3200-3570 \mathrm{~cm}^{-1}$ due to hydrogen bond interactions. These results confirm the interaction between CA and APPPKK. Moreover, the CA characteristic peak were less prominent in CA-APPPKK, compared to the peaks observed in caffeic acid spectrum. ${ }^{26}$

\subsection{Cell viability assay}

Cell viability assays are significant for identifying toxicity by showing the cellular response to a toxic chemical and providing details of cell death, survival, and metabolic activities. The cytotoxicity of the various concentrations of CA, APPPKK and CA-APPPKK (dose range 12.5-100 $\mu \mathrm{M}$ ) on the viability of fibroblast cells was determined by a cell counting kit-8 (CCK-8) assay at $24 \mathrm{~h}$ (Fig. 4a). A significant dose dependent decrease in the percentage of viable cells exposed to CA was observed. Moreover, a previous study reported that some polyphenols of CA (hydroxycinnamate group) can inhibit the growth of fibroblast cells. ${ }^{27}$ These results show that low concentrations of CA have a cytostatic effect, while high concentrations of CA cause cytotoxicity. In contrast, cytotoxicity of APPPKK and CA conjugated with APPPKK, was lower than that with CA alone. When the concentration was improved to $100 \mu \mathrm{M}$, the cell viability was still above $90 \%$ for the CA-APPPKK samples, indicating the low toxicity and excellent biocompatibility of the CA-APPPKK. The results could open an avenue in CA conjugated with APPPKK used as a safe probe for in vivo and biomedical applications. ${ }^{28}$

\subsection{ROS production of CA-APPPKK}

Reactive oxygen species (ROS) are considered signaling molecules participating in many cellular functions, including cell proliferation, differentiation, and apoptosis. To evaluate the endogenous ROS production in the chemical level with CA, APPPKK, and CA-APPPKK, 2',7-dichlorodihydrofluorescein diacetate $\left(\mathrm{H}_{2}\right.$ DCFDA $)$ staining was performed at the chemical level. $\mathrm{H}_{2}$ DCF-DA is an indicator of ROS, which is nonfluorescent until the acetate groups are removed by 
(a)

(c)
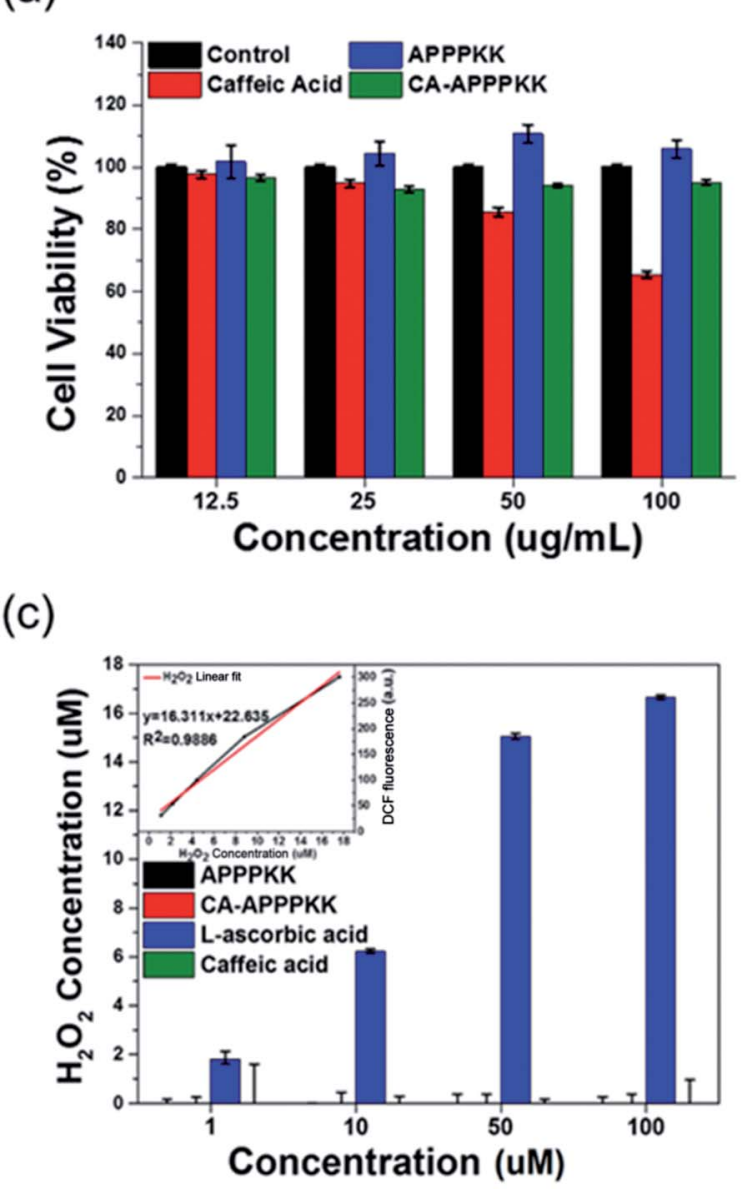

(b)

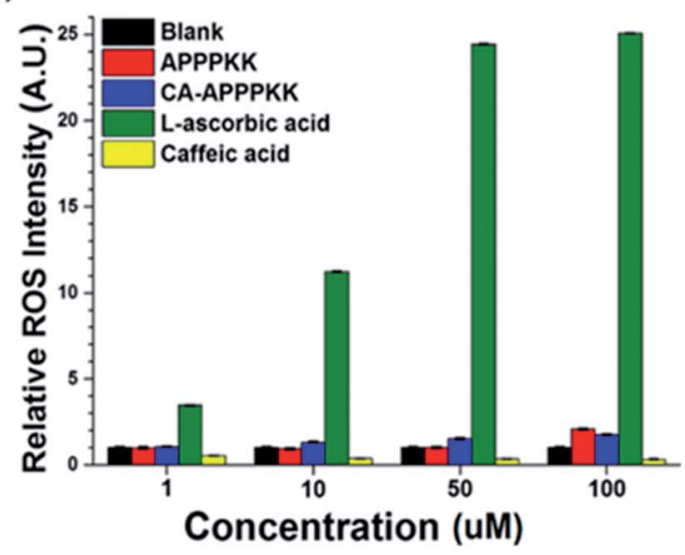

(d)

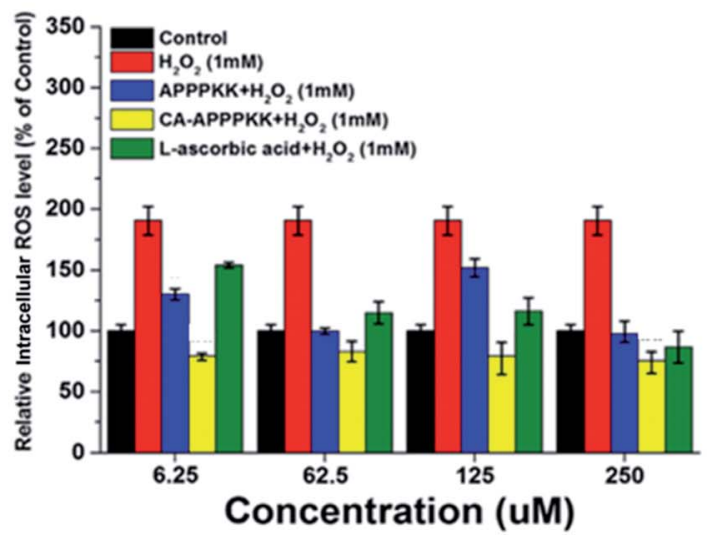

Fig. 4 (a) Cytotoxicity of fibroblast cell treated with APPPKK, CA-APPPKK, and CA at different concentrations (12.5, 25, 50 and 100 $\mu$ M) for $24 \mathrm{~h}$ was determined using the CCK- 8 assay method. (b) ROS generation monitored using APPPKK, CA-APPPKK, and CA at different concentrations $\left(1,10,50\right.$ and $100 \mu \mathrm{M}$ ). (c) Quantification using the standard curve of $\mathrm{H}_{2} \mathrm{O}_{2}$. (d) ROS scavenging monitored using APPPKK, CA-APPPKK, and CA at different concentrations of $(1,10,50$ and $100 \mu \mathrm{M})$ in fibroblast cell.

oxidation. ${ }^{29}$ The ROS level was increased by L-ascorbic acid in a dose-dependent manner (1, 10, 50 and $100 \mu \mathrm{M}$ ) (Fig. 4b). When the concentration was below $100 \mu \mathrm{M}$, the ROS levels induced by L-ascorbic acid were 11 times higher than the CAAPPPKK. Overall, the applied doses of CA, APPPKK and CA$\operatorname{APPPKK}(1,10,50$ and $100 \mu \mathrm{M})$ did not show any obviously ROS generation compared to the control sample. Fig. 4c represents the value of Fig. $4 \mathrm{~b}$ quantitatively from the standard curve of $\mathrm{H}_{2} \mathrm{O}_{2}$. Therefore, CA-APPPKK has potential applications in skin tissue regeneration and wound healing therapeutics.

\subsection{Inhibition of ROS production}

In the present study, the influence of CA, APPPKK, and CAAPPPKK (dose range 6.25-250 $\mu \mathrm{M}$ ) on the intracellular antioxidant enzyme activities of HRP in fibroblast cells with $\mathrm{H}_{2} \mathrm{O}_{2}$ induced oxidative stress was examined to determine if the intracellular ROS scavenging capacities of CA, APPPKK and CAAPPPKK were associated with a modulation of the endogenous antioxidant defense systems. Under normal and oxidative stress conditions, there are sophisticated antioxidant defense systems to eliminate the excess ROS in cells and issues. For example,
HRP converts $\mathrm{H}_{2} \mathrm{O}_{2}$ to $\mathrm{H}_{2} \mathrm{O}$. The biomaterial characteristics can culminate in ROS generation, which is responsible for CAAPPPKK toxicity. The $2^{\prime}, 7^{\prime}$-dichlorodihydrofluorescein diacetate (DCF-DA) techniques were achieved for $24 \mathrm{~h}$ to examine the role of ROS in fibroblast cells with APPPKK, CA-APPPKK, and L-ascorbic acid in the presence of $\mathrm{H}_{2} \mathrm{O}_{2}$ (Fig. 4d). In addition, CA-APPPKK reduced the $\mathrm{H}_{2} \mathrm{O}_{2}$ concentration dramatically after $24 \mathrm{~h}$ at all concentrations, demonstrating that CA (hydroxycinnamate group) in CA-APPPKK undergo $\mathrm{H}_{2} \mathrm{O}_{2}$-mediated oxidation. Surprisingly, the ROS production of CA-APPPKK in fibroblast cells was more pronounced than that of L-ascorbic acid, which was corroborated by the viability results. Moreover, L-ascorbic acid showed higher ROS values than the APPPKK and CA-APPPKK in the presence of $\mathrm{H}_{2} \mathrm{O}_{2}$. Under these experimental conditions, CA-APPPKK can reduce the generation of ROS and exert anti-inflammatory activity in the presence of $\mathrm{H}_{2} \mathrm{O}_{2}$. These results show that CA-APPPKK reacted with overproduced $\mathrm{H}_{2} \mathrm{O}_{2}$ and exerted extremely effective anti-inflammatory activities that reduced or inhibited the larger generation of ROS and cellular damage. Therefore, CA-APPPKK have remarkable ROS scavenger ability as bio-therapeutic agents or upregulated to protect 
the cells from oxidative stress-induced damage and $\mathrm{H}_{2} \mathrm{O}_{2}$ associated diseases.

\subsection{UV protection effect of CA-APPPKK}

To understand the influence of the cytotoxicity on the CAAPPPKK sample, the cellular morphology of the untreated and treated samples was monitored by optical microscopy. The cellular morphological changes were due mainly to UVB radiation. First, HaCaT was treated with $100 \mathrm{~mJ} \mathrm{~cm}^{-2}$ UVB. After UVB irradiation, cellular images were found 12 hours after treatment with CA-APPPKK $(100 \mu \mathrm{M})$ and L-ascorbic acid $(100 \mu \mathrm{M})$, respectively (Fig. 5). Compared to the untreated cells (negative control), the cells treated with UVB showed a significant irradiation-induced decrease in cell viability even after 12 hours of incubation (Fig. 5b). When L-ascorbic acid $(100 \mu \mathrm{M})$ was added to the HaCaT cells promptly after irradiation (Fig. 5c), L-ascorbic acid did not protect against UVB irradiationinhibited cell viability. CA-APPPKK had a protective effect on UVB-induced cell death when added to HaCaT cells promptly after irradiation (Fig. 5d). The CA-APPPKK $(100 \mu \mathrm{M})$ treatment with UVB resulted in decreased cytotoxicity in HaCaT cells compared to that induced by the UVB-irradiation alone or with L-ascorbic acid. These results confirm that CA-APPPKK is less cytotoxic to human skin keratinocytes (HaCaT). Overall, the asprepared CA-APPPKK with valuable biocompatibility could be applied as a favorable biomaterial in biomedical fields, particularly in personal skin-care field.

\subsection{DPPH assay}

The DPPH radical scavenging activity assay is a simple and fast way to determine the antioxidant activity of most bio substances tested in this study. This assay is based on the election transfer
(ET) reaction, which causes the purple DPPH reagent to turn colorless when an antioxidant donates electrons and hydrogen atoms. $^{30}$ The antioxidant activity of L-ascorbic acid, APPPKK, and CA-APPPKK was evaluated using the 'DPPH radical scavenging method'. APPPKK had the lowest DPPH radical scavenging activity of $10.0 \%$, whereas CA-APPPKK had the highest antioxidant activity of $87.3 \%$ at $100 \mu \mathrm{M}$ (Fig. 6a). CA is hydrophobic and insoluble in water, whereas APPPKK is highly soluble. By conjugating CA with APPKK, the composite becomes soluble in water due to the formation of an amide bond between CA and APPPKK through covalent linkage. Also the CA-APPPKK compound will exhibit antioxidant property while adding the antioxidant rich CA with APPPKK. The final product is soluble and showing excellent antioxidant property which is biodegradable and biocompatible. In this study, it is speculated that a maximum CA-APPPKK concentration of $100 \mu \mathrm{M}$ could increase the antioxidant activity, preventing $\mathrm{H}_{2} \mathrm{O}_{2}$-induced DNA damage as well as the generation of lipid peroxidation products, and it could suppress inflammatory reactions in RAW264.7 cells.

\subsection{Long term stability assay}

A significant indicator of the long-term stability of the antioxidant activity (\% scavenging) is the influence of APPPKK, and CA-APPPKK under various storage periods ( 1 to 5 weeks). APPPKK showed the lowest antioxidant ability of $4.89 \%$ for 5 weeks. The antioxidant activity of the CA-APPPKK showed higher antioxidant activity of $87.26 \%$ and $83.69 \%$ respectively, for 5 weeks compared to L-ascorbic acid. Moreover, the resulting vitamin $\mathrm{C}$ showed a gradual decrease in antioxidant activity over 5 weeks, which gave a lower antioxidant ability of $2.89 \%$ (Fig. 6b). The antioxidant activity of the CA-APPPKK remained (a)

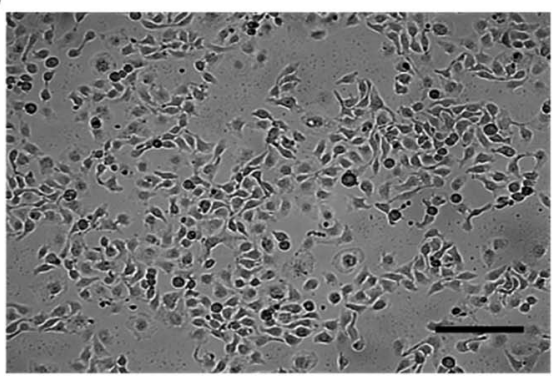

(c)

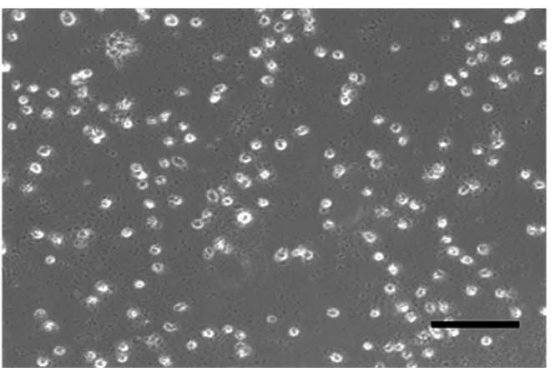

(b)

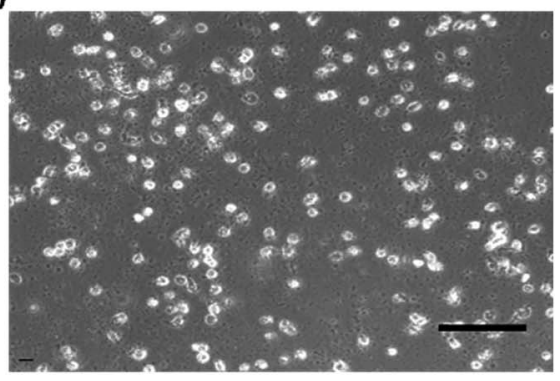

(d)

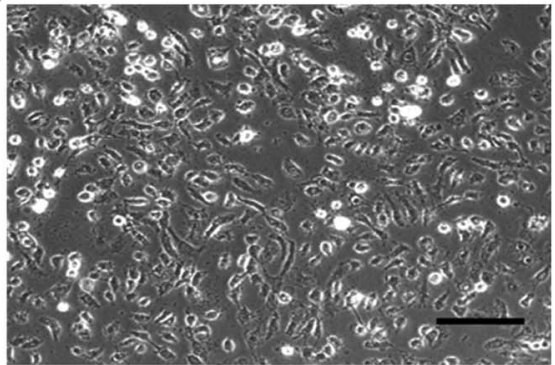

Fig. 5 Cell viability of HaCaT cells (human skin keratinocytes) stimulated with (a) control untreated cells, (b) only UVB, (c) cells treated with 100 $\mu \mathrm{M}$ of L-ascorbic acid, (d) cells treated with $100 \mu \mathrm{M}$ of CA-APPPKK for $24 \mathrm{~h}$; scale bar $=150 \mu \mathrm{m}$. 
(a)

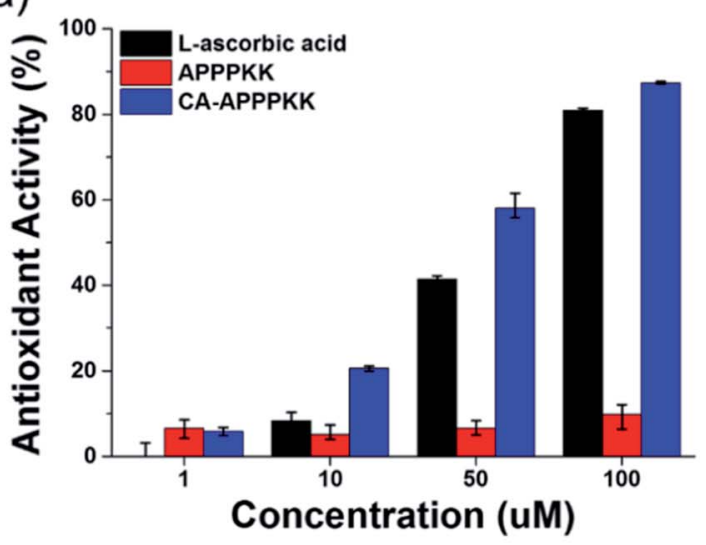

(c)

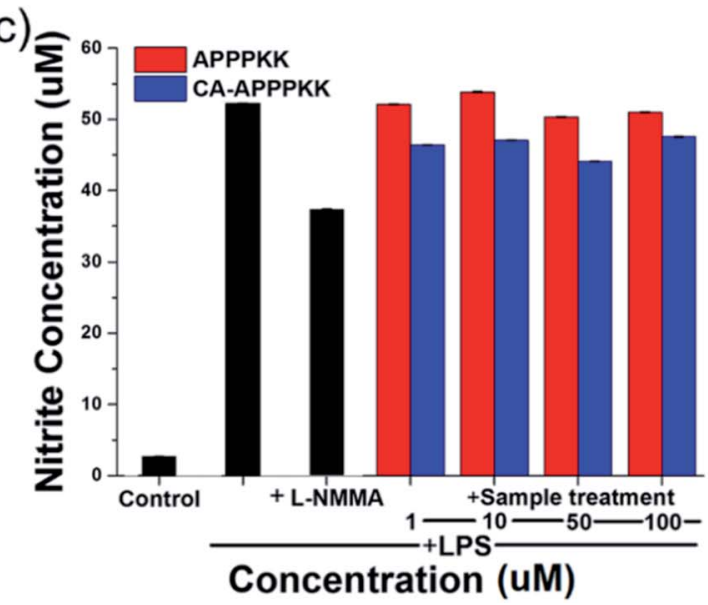

(b)

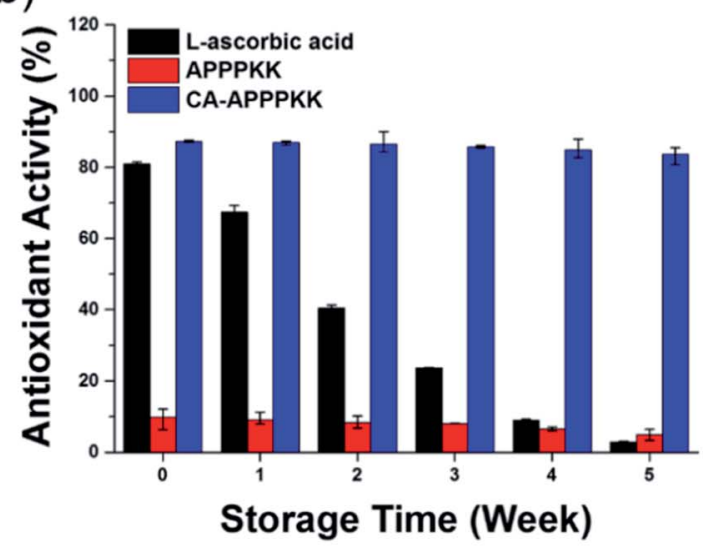

(d)

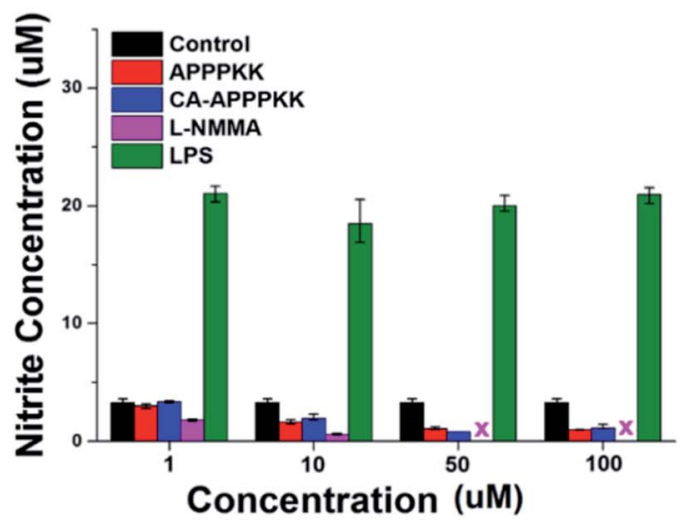

Fig. 6 (a) DPPH radical anti-oxidant activity of different biomaterials at different concentrations of APPPKK, CA-APPPKK, and L-ascorbic acid (1, 10, 50 and $100 \mu \mathrm{M}$ ) for $24 \mathrm{~h}$. (b) Storage stability of the antioxidant activity (\% scavenging) activity of APPPKK, and CA-APPPKK. (c) Antiinflammatory assay (NO assay) assay for various surface functionalized lipopolysaccharide (LPS) L-NMMA, APPPKK, and CA-APPPKK at different concentrations in Raw 264.7 macrophages, respectively. (d) NO production by Raw 264.7 macrophages upon $24 \mathrm{~h}$ treatment with different biomaterials at different concentrations of APPPKK, CA-APPPKK, and L-ascorbic acid $(1,10,50$ and $100 \mu \mathrm{M})$. NO production was measured using Griess assay.

unchanged for 5 weeks at room temperature. Because of the good polyphenol storage stability at this temperature, the CAAPPPKK revealed excellent antioxidant activity, which remained unchanged throughout the 5 weeks of storage. Previous studies correlated the reducing capacity of phenolic compounds to their electron-donating ability. ${ }^{5}$ Therefore, the effective electron (hydrogen) donating ability of the CA-APPPKK is believed to have contributed to the overall observed antioxidant property.

\subsection{NO assay}

Nitrite is a well-known highly toxic substance and bioactive free radical produced during inflammatory events and it plays a key role in several biological and pathological conditions. ${ }^{4}$ The antiinflammatory effect of $N^{\mathrm{G}}$-methyl-L-arginine acetate salt (L-NMMA, dose $20 \mathrm{nM}$ ), APPPKK, and CA-APPPKK (dose range 1-100 $\mu \mathrm{M}$ ) on the production of NO of Raw 264.7 macrophages was determined using a Griess assay at $24 \mathrm{~h}$ (Fig. 6c). The stimulation of RAW264.7 cells with lipopolysaccharide (LPS, dose $1 \mu \mathrm{g} \mathrm{mL}{ }^{-1}$ ) led to a significant increase in the continuous production of NO, which may lead to DNA damage, disruption of DNA repair, and cancer after translational modification. Incubating the cells with L-NMMA inhibited the production of NO in cultured cells. To examine the anti-inflammatory effects of APPPKK, and CA-APPPKK, this study examined whether the LPS-induced inflammatory responses in RAW264.7 cells can be inhibited by CA-APPPKK. The Raw 264.7 macrophages treated with $(1,10,50$ and $100 \mu \mathrm{M})$ of CA-APPPKK showed decreased NO production compared to that of LPS-treated cells. These results also suggest that CA-APPPKK may act as an antiinflammatory agent with a good safety profile for both skin care and wound therapies.

Fig. 6d shows the influence of LPS, L-NMMA, APPPKK and CA-APPPKK (dose range 1-100 $\mu \mathrm{M}$ ) on the inflammatory activity. Interestingly, the results showed that NO production was induced by a treatment with LPS. Among the four biomaterials, LPS was the most powerful, inducing a $600 \%$ increase in nitrite production by the LPS-activated RAW264.7 cells. L-NMMA and APPPKK showed no significant nitrite production 
at different concentrations $(1-100 \mu \mathrm{M})$ in the Raw 264.7 cell. Exposure to $100 \mu \mathrm{M}$ of CA-APPPKK did not cause an inflammatory response and it had a slightly inhibitory effect on the inflammatory response (Fig. 6d), which was maintained at a similar value to that of the control samples. These results indicate that CA-APPPKK, containing phenolic compounds (CA), can scavenge oxygen free radicals and NO radicals by different antioxidant models.

\section{Conclusion}

This paper reported a facile and inexpensive approach for the conjugation of caffeic acid with APPPKK peptide. The fabricated CA-APPPKK was characterized physio-chemically using MALDITOF and HPLC-MS. The cytotoxicity results clearly indicated that CA-APPPKK does not have cytotoxic effects on the human cell line. In addition, CA-APPPKK exhibited interesting dosedependent antioxidant and free radical scavenging activities. CA-APPPKK possesses effective antioxidant activity, which includes free radical and superoxide anion radical scavenging and reducing power. This study investigated ROS generation from various CA-APPPKK surfaces and showed that surface functionalization is the key factor influencing ROS generation and biomaterials toxicity. Such a fabrication approach of CAAPPPKK provides an efficient shape control and cost-effective approach that is potentially competitive for scaling-up to industrial production. This approach highlights the potential of caffeic acid conjugated with АРPРКK in skin care and wound healing applications.

\section{Acknowledgements}

This study was supported by a grant of the Korea Industrial Complex Corp. (KICOX, No. RIC16001). This work was also supported by Inha University WCSL research grant, Korea.

\section{References}

$1 \mathrm{H}$. Wen, X. Gao and J. Qin, Integr. Biol., 2013, 6, 35-43.

2 G. R. Boss and J. E. Seegmiller, West. J. Med., 1981, 135, 434440.

3 D. B. Kim, G. H. Shin, J. M. Kim, Y. H. Kim, J. H. Lee, J. S. Lee, H. J. Song, S. Y. Choe, I. J. Park, J. H. Cho and O. H. Lee, Food Chem., 2016, 194, 920-927.

4 R. P. Singh, S. Sharad and S. Kapur, J. Indian Acad. Clin. Med., 2004, 5, 218-225.

5 Y. C. Hseu, W. H. Chang, C. S. Chen, J. W. Liao, C. J. Huang, F. J. Lu, Y. C. Chia, H. K. Hsu, J. J. Wu and H. L. Yang, Food Chem. Toxicol., 2008, 46, 105-114.

6 V. S. Chedea, L. Choueiri, M. Jisaka and P. Kefalas, Food Chem., 2012, 135, 1999-2004.
7 N. Oršolić, M. Kunštić, M. Kukolj, R. Gračan and J. Nemrava, Chem.-Biol. Interact., 2016, 256, 111-124.

8 A. T. E. Vilian, S.-M. Chen, Y.-H. Chen, M. A. Ali and F. M. A. Al-Hemaid, J. Colloid Interface Sci., 2014, 423, 33-40.

9 İ. Gülçin, Toxicology, 2006, 217, 213-220.

10 J. H. Chen and C. T. Ho, J. Agric. Food Chem., 1997, 45, 23742378.

11 M. G. Ignatova, N. E. Manolova, I. B. Rashkov, N. D. Markova, R. A. Toshkova, A. K. Georgieva and E. B. Nikolova, Mater. Sci. Eng., C, 2016, 65, 379-392.

12 A. A. Lozano-Pérez, H. C. Rivero, M. d. C. Pérez Hernández, A. Pagán, M. G. Montalbán, G. Víllora and J. L. Cénis, Int. J. Pharm., 2017, 518, 11-19.

13 Y. Sato, S. Itagaki, T. Kurokawa, J. Ogura, M. Kobayashi and T. Hirano, Int. J. Pharm., 2011, 403, 136-138.

14 Y. Li, F. Jiang, L. Chen, Y. Yang, S. Cao, Y. Ye, X. Wang, J. Mu, Z. Li and L. Li, FEBS Open Bio, 2015, 5, 466-475.

15 M. F. Tolba, H. A. Omar, S. S. Azab, A. E. Khalifa, A. B. AbdelNaim and S. Z. Abdel-Rahman, Crit. Rev. Food Sci. Nutr., 2016, 56, 2183-2190.

16 S. Yu, H. Hsieh, J. Pang, D. Tang, C. Shih and M. Tsai, Food Hydrocolloids, 2013, 32, 9-19.

17 G.-W. Oh, S.-C. Ko, J.-Y. Je, Y.-M. Kim, J. Oh and W.-K. Jung, Int. J. Biol. Macromol., 2016, 93, 1549-1558.

18 T. T. Cao and Y. Q. Zhang, Mater. Sci. Eng., C, 2016, 61, 940952.

19 M. Yan, J. Du, Z. Gu, M. Liang, Y. Hu, W. Zhang, S. Priceman, L. Wu, Z. H. Zhou, Z. Liu, T. Segura, Y. Tang and Y. Lu, Nat. Nanotechnol., 2010, 5, 48-53.

20 L. Yang, Z. Zheng, C. Qian, J. Wu, Y. Liu, S. Guo, G. Li, M. Liu, X. Wang and D. L. Kaplan, J. Colloid Interface Sci., 2017, 496, 66-77.

21 M. V. Blagosklonny, Drug Discovery Today, 2007, 12, 218-224.

22 S. J. Green, M. S. Meltzer, J. B. Hibbs and C. A. Nacy, J. Immunol., 1990, 144, 278-283.

23 Z. Liu and K. L. Schey, J. Am. Soc. Mass Spectrom., 2005, 16, 482-490.

24 S. M. Mandal and S. Dey, J. Biomol. Tech., 2008, 19, 116-121. 25 Y. Xing, H. Peng, M. Zhang, X. Li, W. Zeng and X. Yang, J. Zhejiang Univ., Sci., B, 2012, 13, 487-493.

26 A. Belay, H. K. Kim and Y.-H. Hwang, Luminescence, 2016, 31, 565-572.

27 J. M. Glamoclija, J. Sci. Food Agric., 2013, 93, 3205-3208.

28 T. Arai, A. Ohno, K. Mori, H. Kuwata, M. Mizuno, K. Imai, S. Hara, M. Shibanuma, M. Kurihara, N. Miyata, H. Nakagawa and K. Fukuhara, Bioorg. Med. Chem. Lett., 2016, 26, 5468-5471.

29 I. Rosenthal and E. Ben-Hur, Int. J. Radiat. Biol., 1995, 67, 8591.

30 D. B. Kim, G. H. Shin, Y. J. Lee, J. S. Lee, J. H. Cho, S. O. Baik and O. H. Lee, Food Chem., 2014, 151, 58-64. 